\title{
The structure and function of the epidermal barrier in patients with atopic dermatitis - treatment options. Part two
}

\author{
Jagoda Pelc, Magdalena Czarnecka-Operacz, Zygmunt Adamski \\ Department of Dermatology and Venereology, Poznan University of Medical Sciences, Poznan, Poland \\ Adv Dermatol Allergol 2018; XXXV (2): 123-127 \\ DOI: https://doi.org/10.5114/ada.2018.75234
}

\begin{abstract}
Atopic dermatitis (AD) is a chronic and recurrent disease induced by underlying defects of the epidermal barrier and immunological disorders, typical of atopic diseases. The genetic and immunological mechanisms (outlined in the previous paper) affecting the dysfunction of the barrier are intensified by environmental factors, e.g. airborne and food allergens, infections and stress. For this reason, proper skin care, which prevents further damage and restores the epidermal barrier is of such importance in the field of AD therapy. Appropriate therapy is based on emollients which, coupled with anti-inflammatory and antipruritic treatment, should be used as the first-line therapy. The aim of the present paper is to outline the effects of the abovementioned factors on the dysfunction of the epidermal barrier as well as to emphasize the importance of proper atopic skin care in maintaining the integrity of the barrier and preventing exacerbation of the disease.
\end{abstract}

Key words: atopic dermatitis, epidermal barrier, therapy, emollients.

\section{Immunological facets of epidermal barrier dysfunction in atopic dermatitis: effects of IgE antibodies}

As has been mentioned previously [1], 80\% of atopic dermatitis (AD) sufferers show elevated serum IgE levels, IgE-dependent allergic response to airborne and food allergens as well as a higher risk of developing clinical signs of allergic rhinitis, allergic conjunctivitis and asthma. Those patients exhibit marked tissue eosinophilia and intensified tissue release of post-inflammatory cytokines [2]. In the remaining $20 \%$ of sufferers from AD while presenting normal levels of serum IgE concentration, the onset of AD symptoms is usually observed at a later age (over 20) and the IgE-dependent allergic reaction to classical environmental allergens does not appear. However, it is currently considered crucial that some of those patients develop an allergic reaction to bacterial antigens, yeast-like fungi (Staphylococcus aureus, Candida albicans, Pityrosporum ovale enterotoxins) or autoantigens (autoreactivity). Additionally, children present transient $A D$ with initial low serum concentration of IgE and devoid of the typical hypersensitivity which only develops later (usually over the age of 5), in relation to the defec- tive structure and function of the epidermal barrier [1, 3]. It is obviously known that antigen-specific IgE antibodies (aslgE) do indeed play a vital role in the development of skin inflammation through classic activation of mast and dendritic cells [2]. As mentioned above, some AD patients show aslgEs directed against microbial antigens present on the surface of the epidermis (e.g. S. aureus, Malassezia spp. and Trichophyton rubrum), and against autologous human proteins (e.g. epidermal autoantigen Hom s1). It is known that some environmental antigens are similar in structure to the abovementioned proteins. The cross-binding of autoantigens and aslgE antibodies results in a highly acute inflammation process [4], while IgE directed against human autoantigens may cause type I hypersensitivity and stimulate dendritic cells, inducing proliferation of auto-reactive T cells [1].

\section{Epigenetic facets of defective structure and function of the epidermal barrier}

In AD, skin shows higher sensitivity to external and internal factors alike in comparison to the healthy population. Environmental allergens, high stress levels, insufficient hydration, hyperhidrosis, irritants (wool, acrylic,

Address for correspondence: Jagoda Pelc MD, Department of Dermatology and Venereology, Poznan University of Medical Sciences, 49 Przybyszewskiego St, 60-355 Poznan, Poland, phone: +48 667261 626, e-mail: jagmos@wp.pl Received: 18.04.2017, accepted: 21.06.2017. 
soaps, detergents) may exacerbate itchiness and may lead to excessive scratching related to the itching sensation [5].

Atopic dermatitis risk factors include exposure to aeroallergens (animal epidermis and hair, plant pollens, house dust mites). Such exposure may also be a factor in the exacerbation of AD in adult patients [1]. House dust mites are a source of 30 different proteins inducing IgE-dependent reaction, including cysteine and serine proteases [6]. The development of AD in children is also affected by hypersensitivity to food allergens [1]. Children with food allergies show positive immediate reaction in skin tests or presence of serum lgE antibodies directed against various foods (mostly eggs, milk, wheat, soy and peanuts). T cells directed against food allergens isolated from skin lesions of $A D$ patients prove that certain foods may induce an immunological response of the skin. Food may exacerbate the symptoms of AD through allergic and non-allergic hypersensitivity reactions [1]. Over the age of 3, children "grow out" of food allergies, while tolerance is being developed, but hypersensitivity to airborne allergens is still relatively common. Obviously exposure to hose allergens may lead to the exacerbation of inflammatory skin lesions and itchiness. In atopy patch tests (APTs), and it has been proven that application of atopic allergens on the epidermis of lesion-free skin areas causes eczematous reaction in the allergic type of $A D$ patients. The effect of aeroallergens on the immunological response in the atopic skin is evidenced by the isolation of T cells directed against Dermatophagoides pteronyssinus (Dp) and other aeroallergens from skin lesions of patients with $A D$ [1].

Stimulation of the immunological system induced by stress factors is altered in AD patients, although the exact mechanisms underlying this process are not known. These patients exhibit higher levels of the nerve growth factor (NGF) and substance P (SP), which may correlate with the activity of the disease. Higher levels of brain derived growth factor in AD sufferers result in reduced apoptosis of eosinophils, which in turn intensifies the invitro chemotaxis of eosinophils [1].

Coarse and wooly clothing often leads to mechanical irritation and exacerbation of AD. Other irritants such as chemical substances used to wash the skin should also be avoided [1]. Soaps and detergents dissolve epidermal lipids. Furthermore, through raising the $\mathrm{pH}$ levels, they enhance the activity of proteases and thereby elicit the release of cytokines by corneocytes [7]. Using hard and hot water to wash the body also has an adverse effect [6]. Etiopathogenesis also mentions the effects of environmental pollution, food preservatives and early introduction of allergenic foods into children's diets. The role of exposure to cigarette smoke in the development of $A D$ is disputable [1].

It has been proven that glucocorticosteroids (GCS) have a negative influence on the epidermis as they in- hibit the synthesis of ceramides, cholesterol and free fatty acids (by inducing expression of kallikrein 7) thin the stratum corneum and reduce the anti-bacterial properties of epidermis [7].

Most AD sufferers exhibit $S$. aureus skin colonization, which may exacerbate the inflammation. Deficiencies of antimicrobial proteins (AMPs) facilitate colonization and development of infections as well as exacerbation of the inflammatory process. Expression of defensins (antibacterial and anti-fungi antibiotics) is limited by cytokines (IL-4 and IL-13) in the skin of AD sufferers [2]. The defect of the immunological system, including the reduction of antibacterial proteins, reduced neutrophil recruitment, Toll-like receptor (TLR) defect, as well as the defect of the epidermal barrier play a pivotal role in bacterial colonization and infections in AD. HBD-3 mobilization and eradication of S. aureus by keratinocytes are hindered considerably by IL-4 and IL-13. Atopic dermatitis sufferers produce aslgE antibodies directed against toxins. Basophils release histamine as a result of the exposure to toxins. This indicates that $S$. aureus superantigens (mostly enterotoxins A and $B$ ), i.e. the toxic substances, may cause mast cells to degranulate in response to the penetration of the epidermal barrier and may thus lead to itchiness and acute inflammation. Staphylococcus aureus causes skin lesions in AD through activating T-lymphocytes with superantigens, through induction of proinflammatory cytokines and chemokines via TLR receptors and through inducing cytotoxicity of $\alpha$-toxin to keratinocytes [8]. Superantigens also induce immunity to glucocorticosteroids, which impedes response to treatment. The binding of S. aureus to the skin is strengthened by skin inflammation in AD. Components of the cell wall and the $S$. aureus cell envelope are simply regarded to be allergens; they are capable of releasing histamine directly from basophils and mast cells, of inhibiting synthesis of IgG and IgA as well as of intensifying the synthesis of IgE [9]. Steroid or tacrolimus (calcineurin inhibitor) treatment reduces the amount of $S$. aureus in terms of colonization of AD skin. Lowered levels of pro-inflammatory cytokines (TNF- $\alpha$ and IFN- $\gamma$ ) make the skin more prone to infection [1]. One factor which facilitates bacterial colonization in AD may be the altered composition of lipids and fatty acids in the skin, e.g. lower ceramide content and higher amount of cholesterol in the stratum corneum. Additionally, bacterial colonization in $A D$ is fostered by the altered composition of the fatty acids in the sebum which covers the skin of the sufferers [9]. Bourrain et al. evaluated the composition of microflora (using molecular biology techniques) in AD sufferers on the healthy as well as inflamed skin areas before commencing the treatment and later, during hydrotherapy. They noted dominance of pathological flora in the inflamed areas and restoration of diverse commensal flora during the treatment [8]. Although S. aureus is prevalent on the damp areas of the skin (elbows and knees are typical areas for AD lesions), hydrotherapy and the use of moisturizing products may 
result in the growth of commensal microflora, which impedes colonization by S. aureus [8]. Staphylococcus aureus is detected in the skin of about $90 \%$ of AD patients. It induces keratinocytes and immunological system cells to secrete agents causing the dysfunction of the epidermal barrier. Staphylococcus aureus impedes the final differentiation of keratinocytes by stimulating IL- 6 secretion, which in turn, reduces the expression of keratin 1 and keratin 10 in keratinocytes. Abnormal keratinization may result in the reduced expression of filaggrin. Moreover, S. aureus is probably involved in the transepidermal water loss (TEWL) increase, lowered hydration, skin dryness and increase in peeling [10]. It releases numerous proteolytic enzymes which damage the epidermal cells and facilitate bacterial penetration. Alkaline skin pH (7.0-8.0) creates a conducive environment for adhesion of S. aureus to keratinocytes [9]. This bacterium also disrupts the integrity of the epidermal barrier by releasing enzymes which impede ceramide synthesis (glucosylceramide deacylase and sphingomyelin deacylase) [7].

Apart from bacterial infections, the progression of $A D$ may also be exacerbated by viral infections (herpes, warts, molluscum contagiosum) [5]. Greater susceptibility to those infections is related to the disorder of cellular immunity in AD, i.e. deficiency of Th1 lymphocytes and reduced IFN- $\gamma$ production. This is also confirmed by studies which showed a higher incidence of atopic diseases in people who did not experience infectious diseases in their childhood; this is connected to the stimulation of Th1 response in viral diseases, which also translates into impeding Th2 response.

A considerable body of evidence for the role of opportunistic Malassezia yeast in the process of the epidermal barrier dysfunction has surfaced recently. Several studies have shown the presence of specific IgE in the serum and positive results of skin prick tests as well as atopy patch tests for Malassezia in adults with AD [1]. Patients with inflammation in the head and neck area often exhibit Malassezia species colonization [2, 11]. IgEdependent allergic reaction to Malassezia is specific to $A D$ patients, but not found in patients with asthma and ANN [1]. First records of the relationship between AD and Malassezia come from 1983 (Clemmensen and Hjorth). They demonstrated that ketoconazole treatment relieves eczematous lesions in patients with positive results of Malassezia SPTs. The Malassezia yeast belong to the natural skin flora and colonize the stratum corneum, hair follicles and, primarily, skin areas rich in sebaceous glands. Despite that, Malassezia species may cause skin infections or even systemic infections. It is the causative factor behind Tinea versicolor and it plays an important role in the pathogenesis of seborrheic dermatitis (mainly M. globosa, M. furfur, M. sympodialis) and atopic dermatitis. Malassezia may impair the functioning of the epidermal barrier. Both the cells and allergenic components of the yeasts, crossing the skin barrier, connect with the Langerhans cells in the epidermis. Internalization of Malassezia is connected with maturation of monocyte-derived dendritic cells and production of proinflammatory cytokines (TNF- $\alpha$, IL-1 $\beta$, IL-18). As a result of microbiological stimulation, dendritic cells produce IL-2, which is necessary for appropriate activation of T cells. The presence of aslgE antibodies directed against Malassezia in the serum of AD patients was first described in 1990. The frequency of their incidence in AD patients ranges from $32 \%$ to $68 \%$, while $30-80 \%$ of AD patients have positive results of Malassezia SPTs. Atopic dermatitis patients with no symptoms on their heads or necks as well as patients with low serum IgE levels were found to have positive results of atopy patch tests for Malassezia. Positive APT results were also found in patients with non-detectable aslgE levels and, in some cases, also in patients with negative results of Malassezia SPTs. Those reactions may depend on the small amounts of aslgE, not detected by SPTs, but sufficient for antigen presentation. The allergy most likely occurs in the skin barrier where the Langerhans cells interact with Malassezia-specific antigens, migrate to local lymph nodes and present allergens to other cells in the memory-creating component of the immunological system [11].

\section{Improvement of the structure and function of the epidermal barrier in AD sufferers should constitute main therapy goal}

Patients are advised to implement an appropriate atopic skin care routine, which restores the damaged epidermal barrier and thus reduces the risk of microbial and allergen penetration. Atopic skin care should be based on emollients, which moisturize the skin, prevent dryness, relieve itchiness and inflammation and prevent irritants from entering the skin, thus improving the structure and functioning of the epidermal barrier. Their importance for preventing the development of AD symptoms was demonstrated by British researchers, who used them in newborns with a high risk of developing AD [12]. Likewise, a similar study conducted in Japan also confirmed the validity of using emollients in infancy to reduce the risk of $A D$ development [13]. Baths with the use of emollients should not be longer than $10 \mathrm{~min}$ and the water temperature should not be higher than $36^{\circ} \mathrm{C}$. Following the bath, skin should be dried gently, without rubbing, with emollients applied directly afterwards.

One should absolutely avoid skin-irritating factors such as wearing clothes made from irritating synthetic materials or wool, using irritating soaps and hot, hard water for baths. Cleansers with $\mathrm{pH}$ range of 5.5-6.0 should be used as they maintain acidic skin environment. It is also advisable to consider eliminating potentially harmful aeroallergens and food allergens as well as to avoid bacterial and viral infections (as mentioned above, microorganisms may act as super-antigens). The risk of developing AD in child- 
hood may be reduced through breastfeeding for a minimum of 4 months but this solution is short-termed and only effective until the age of 3 [1].

The progression of $A D$ is also influenced by vitamin $D$; however, its bearing on the pathogenesis of the disease remains highly controversial. Vitamin D increases the amount of anti-bacterial peptides and plays a role in the regulation of the immunological response [14]. Its antiinflammatory and immunomodulatory properties depend on its proper concentration in the body; it appears that the beneficial concentration of calcidiol in the blood serum falls within the range of $30-40 \mathrm{ng} / \mathrm{ml}$. The active form of vitamin $D$ (calcitriol), produced by keratinocytes irrespective of the liver and kidney function, regulates the proliferation and differentiation of cells and thus preserves the integrity of the epidermal barrier [15].

Inflammation and the following activation of the immunological system impairs the functioning of the epidermal barrier in AD [14]; thus, apart from atopic skin care, anti-inflammatory therapy is of vital importance. The therapy mostly utilizes topical products (calcineurin inhibitors and glucocorticosteroids.)

Topical calcineurin inhibitors (tacrolimus ointment, pimecrolimus cream) selectively inhibit the activation of $T$ cells and mast cells and the production of pro-inflammatory cytokines (IL-31 produced by Th2 lymphocytes is a vital pruritus mediator). Thus, they exhibit pruritus-relieving properties; in the case of pimecrolimus, the relief is observed within $48 \mathrm{~h}$ from the implementation of treatment in clinical trials. They do not cause skin atrophy or other complications typical of glucocorticosteroids. They restore the epidermal barrier through influencing gene expression. Pimecrolimus may reinforce the expression of anti-bacterial proteins in keratinocytes and enhance their ability to curb S. aureus growth [16]. Tacrolimus has stronger anti-inflammatory properties than pimecrolimus; moreover, it impairs the functioning of Langerhans cells. Proactive tacrolimus therapy is advisable, coupled with complementary emollient therapy [2].

Atopic dermatitis therapy employs glucocorticosteroids with the lowest possible potencies and the shortest possible treatment duration, due to their detrimental ef fects on the epidermis, as mentioned above. Combining topical steroid therapy with emollients accelerates the process of restoring the epidermal barrier and allows for earlier dosage reduction. Furthermore, emollients bind free glucocorticosteroid particles in intercellular spaces, thus reducing the risk of adverse effects of steroids.

In AD anti-pruritus treatment, first- and second-generation antihistamines are used alongside appropriate skin care and anti-inflammatory treatment, with second-generation antihistamines showing fewer adverse effects [2].

In some cases, systemic therapy, such as phototherapy or other immunosuppressive therapy methods (cyclosporine A, azathioprine, methotrexate, mycophenolate mofetil, biological therapies: dupilumab), are necessary.
Oral steroids are generally not recommended due to their adverse effects [17]. In exceptional cases they may be used as a short-term solution (from 7 to 14 days).

Due to a higher risk of skin superinfection and extensive colonization by $S$. aureus in AD sufferers, antimicrobial therapy is also administered, both topically and systemically. The possible topical therapy options include triclosan, chlorhexidine and antibiotics (fusidic acid, erythromycin). Systemic therapy with antibiotics is used in high-incidence secondary bacterial infections (mostly S. aureus). First- and second-generation cephalosporins and half-synthetic penicillins are used for a period of 7 to 10 days [1]. In the case of immunity to erythromycin, macrolides provide an alternative therapy option, whilst in the case of allergy to penicillins or cephalosporins, clindamycin or fusidic acid are administered orally. In the case of AD combined with eczema herpeticum (EH), general antiviral therapy is used (acyclovir, valaciclovir). Eczema herpeticum risk factors include early AD onset, acute and untreated $A D$, dermatitis in the head and neck area, previous EH and HSV infections, heightened total IgE levels [18].

In the case of fungal infections, appropriate antifungal therapy is employed. Improvement of the skin condition was noted following antifungal therapy with ketoconazole, which indicates the probable presence of M. furfur [5].

\section{Summary}

Maintaining the integrity of the epidermal barrier is a key factor in preventing the symptoms of $A D$ and in alleviating their exacerbation. The problem of AD concerns both structural and functional defects of the barrier; it is connected with the influence of many internal as well as environmental factors. Thus, a multi-directional approach to therapy is necessary. Maintaining the structure and functioning of the epidermal barrier should be given special care as early as in the first months of life, especially in the case of children with a family history of atopic diseases. Emollients are singled out of as the first-choice option among the numerous prevention and therapy solutions. It ought to be remembered that emollients should constitute a sort of 'basis' and be combined with other therapy methods, as mentioned above, in line with particular needs. Appropriate skin care applied from the moment of birth is vital for success in the fight against AD.

\section{Conflict of interest}

Professor Magdalena Czarnecka-Operacz MD, PhD: training courses, lecturing (post-graduate training), (Almiral, Astellas, Meda, Berlin Chemie, Novartis), member of the Skin Academy and Advisory Board (Almiral), clinical trals (phase 2, 3) and Scientific projects (Almiral, 
Astellas, Meda, Berlin Chemie, Novartis). Others authors declare no conflict of interest.

\section{References}

1. Akdis CA, Akdis M, Bieber T, et al. Diagnosis and treatment of atopic dermatitis in children and adults: European Academy of Allergology and Clinical Immunology/American Academy of Allergy, Asthma and Immunology/PRACTALL Consensus Report. Allergy 2006; 61: 969-87.

2. Silny W, Czarnecka-Operacz M, Gliński W, et al. Atopowe zapalenie skóry - wspótczesne poglądy na patomechanizm oraz metody postępowania diagnostyczno-leczniczego. Stanowisko grupy specjalistów Polskiego Towarzystwa Dermatologicznego. Post Dermatol Alergol 2010; 27: 365-83.

3. Novembre E, Cianferoni A, Lombardi E, et al. Natural history of "intrinsic" atopic dermatitis. Allergy 2001; 56: 452-3.

4. Ring J, Darsow U. Atopowe zapalenie skóry. In: Braun-Falco Dermatologia. Burgdorf WHC, Plewig G, Wolff HH, et al. (eds.). Czelej, Lublin 2010; 425-41.

5. Leung DYM. Pathogenesis of atopic dermatitis. J Allergy Clin Immunol 1999; 104: 99-108.

6. Cork MJ, Danby SG, Vasilopoulos Y, et al. Epidermal barrier dysfunction in atopic dermatitis. J Invest Dermatol 2009; 129: 1892-908.

7. Samochocki Z. Wpływ zaburzeń bariery naskórkowej na rozwój i przebieg atopowego zapalenia skóry. Alergia 2010; 4: 19-24.

8. Bourrain M, Ribet V, Calvez A, et al. Balance between beneficial microflora and Staphylococcus aureus colonisation: in vivo evaluation in patients with atopic dermatitis during hydrotherapy. Eur J Dermatol 2013; 23: 786-94.

9. Adamek-Guzik T, Guzik T, Czerniawska-Mysik G, et al. Znaczenie obniżonej odporności na infekcje w patogenezie atopowego zapalenia skóry: rola Staphylococcus aureus. Alergia Astma Immunologia 2001; 6: 169-79.

10. Son ED, Kim HJ, Park T, et al. Staphylococcus aureus inhibits terminal differentiation of normal human keratinocytes by stimulating interleukin-6 secretion. J Dermatol Sci 2014; 74: 64-71.

11. Scheynius A, Johansson C, Buentke E, et al. Atopic eczema/ dermatitis syndrome and Malassezia. Int Arch Immunol 2002; 127: 161-9.

12. Simpson EL, Chalmers JR, Hanifin JM, et al. Emollient enhancement of the skin barrier from birth offers effective atopic dermatitis prevention. J Allergy Clin Immunol 2014; 134: 818-23.

13. Horimukai K, Morita K, Narita M, et al. Application of moisturizer to neonates prevents development of atopic dermatitis. J Allergy Clin Immunol 2014; 134: 824-30.

14. Leung DYM. New insights into atopic dermatitis: role of skin barrier and immune dysregulation. Allergol Int 2013; 62: 15161.

15. Pawlak J, Doboszyńska A. Witamina D w chorobach alergicznych. Postepy Hig Med Dosw (online) 2014; 68: 1152-70.

16. Luger T, De Raeve L, Gelmetti C, et al. Recommendations for pimecrolimus $1 \%$ cream in the treatment of mild to moderate atopic dermatitis: from medical needs to a new treatment algorithm. Eur J Dermatol 2013; 23: 758-66.

17. Tollefson MM, Bruckner LA; Section On Dermatology. Atopic dermatitis: skin-directed management. Pediatrics 2014; 134 : e1735-44.
18. Boguniewicz M, Leung DYM. Atopic dermatitis: a disease of altered skin barrier and immune dysregulation. Immunol Rev 2011; 242: 233-46. 